\title{
Los continuum para la inteligencia colectiva desde la coexistencia universidad, redes sociales ${ }^{*}$
}

\author{
Ingrid Nederr Donaire
}

Recibido: 5 de noviembre de 2010 Revisado: 31 de enero de 2011 Aprobado: 10 de febrero de 2011

\section{RESUMEN}

El propósito de este estudio es generar una reflexión sobre la gestión de la coexistencia universidad-redes sociales, como vía de desarrollo del espacio interactivo y de colaboración espontánea que se requiere para la transformación social. Ésta desde la preeminencia de una morfología reticular fundada en la hipermediación tecnológica para la inteligencia colectiva, partiendo del hecho que en el contexto de las sociedades contemporáneas no se constituye sólo por el territorio geográfico, sino que incluye un flujo-reflujo de saberes que potencia y transforma las cualidades de ser y actuar en sociedad. Así, se crea un espacio de coexistencias diversas donde la humanidad se encuentra y se produce a sí misma en una temporalidad complejizante de orden transversal, plural e indeterminada. De allí que admite y privilegia una trama de religues o continuum entre la discontinuidad y continuidad de estados que se prolongan más allá de ellos para remitir a otros donde la ciclicidad reflexiva hace eco y percusión de forma multidimencional.

\section{Palabras clave}

Continuum, coexistencia universidad-redes sociales, inteligencia colectiva, gestión, hipermediación tecnológica y generatividad reflexiva. 


\title{
The Continuum for Collective Intelligence from University Social Coexistence
}

\author{
Ingrid Nederr Donaire
}

\begin{abstract}
The purpose of this study is to create a reflection on management of the coexistence university social networking as a way of development of interactive space and spontaneous collaboration that is required for social transformation from the predominance of a morphology based on grid technology hypermediation for collective intelligence, based on the fact that in the context of contemporary societies it is not only constituted by geographical area, but includes a flow-reflux of knowledge that enhances and transforms the qualities of being and acting in society, creating a space of diverse coexistences, where humanity is found and produces itself in a complexifying temporality plural and which is indeterminate. Hence, it admits and favors a frame or continuum relegated between discontinuity and continuity of states that extend beyond themselves to refer to others where reflective periodicity echoes and strikes in a multidimensional manner many dimensions.
\end{abstract}

\section{KeYWORDS}

Continuum, university-social networks coexistence, collective intelligence, management, technological hypermediation reflective generativity. 


\section{Prólogo}

Muchas son las circunstancias, acciones y relaciones que intervienen en el acontecer contemporáneo y más aún cuando la sociedad implica la existencia de organizaciones, grupos e individuos capaces de pensar, comunicarse, reflexionar y de actuar en consecuencia, es así que toda esta multiplicidad se constituye en una sistematicidad de componentes recíprocamente relacionada con un todo, la cual se instituye y transforma multidireccionalmente.

En tal sentido, gira esta indagación, la cual trata de comprender y destacar la capacidad reflexiva-recursiva que de manera natural moviliza el mundo en devenir, por ello encausa su direccionalidad al ámbito universitario y cómo éste puede aprovechar lo que conlleva esta reflexividad permanentemente cargada de conocimientos nuevos y de una cultura que se redefine continuamente como manera diferente y emergente de percepción y aprehensión para lo cual surgen progresivamente técnicas novedosas soportadas en dispositivos, instrumentos y lenguajes que configuran nuevas pautas de comportamiento como patrones que van remodelando con su resonancia todos los rincones de la sociedad.

Toda esta sistematicidad implica cambios y transformaciones que exigen grandes esfuerzos de adaptación, ya que hacen eco de metamorfosis continuas y profundas, al mismo tiempo que se plantea la búsqueda de situaciones, de certezas que permitan su delimitación y sometimiento. Existe la paradoja a este respecto, pues cuando se presentan cambios graduales se pueden diferenciar con claridad las posiciones extremas; el principio y el fin, pero no se puede precisar el límite donde se produce el cambio de una posición a otra, existe por lo tanto un umbral indefinible, es decir, un continuo en el que las variaciones resultan inapreciables, $\mathrm{o}$ una existencia en la cual una pequeña diferencia determina la regulación o la decisiva transformación.

Por consiguiente, es evidente que esta existencia no se constituye en una particularidad, en un ser único, exclusivo, individual, sino en algo imperceptible, discontinuo e incierto, intrínseco a una temporalidad espacial fundada por una reciprocidad de componentes e interrelaciones de características multireferenciales y heterogéneas.

La noción de continuum admite y privilegia la coexistencia multidimensional, donde en el religue entre la discontinuidad y continuidad de ordenes $u$ estados se prolonga más allá de ellos, para remitir a otros estados, donde la ciclicidad reflexiva hace repercusión por la confluencia de sus autonomías y dependencias. Por ello, tratar de propender a un estado único, es negar la relación indisociable que existe de manera natural, recursiva y recíproca, entre el todo y las partes constituyentes.

Esto encaja perfectamente con la consideración de Deleuze y Guattari (2000) en cuanto a la analogía configurativa de los rizomas, los cuales son tubérculos de tallo horizontal de donde emergen raíces, raicillas, tallos y hojas entretejidas, donde no se conoce su origen y culminación, ya que existe una multidimensionalidad relacionada. De esta manera, la interconexión que se traza en este trabajo permite su abordaje desde cualquier continuum, esto es porque su recorrido va y viene haciendo interferencia constructiva, tal como el principio físico, propio de la 
naturaleza ondulatoria de la luz, donde las ondas en fase se refuerzan mutuamente y el resultado es una onda de mayor amplitud.

De esta manera, la sinergia que se establece de principio a fin en este compendio de saberes no sólo permite establecer multiples vías de conexión para su abordaje, sino que también refleja la complementariedad de dialógicas en una red de generatividad permanente que supera la interrupción en cualquier parte. Al mismo tiempo plantea la equifinalidad de recomenzar desde cualquier interrelación.

Es así que el propósito y alcance de este trabajo es presentar una reflexión sobre los hallazgos y revelaciones investigativas que emergen a partir de las interrelaciones dialógicas inmersas en la praxis universitaria en referencia a lo que implica la hipermediación tecnológica en la configuración de entornos cognitivos abiertos y distribuidos. También lo que estos revierten en los procesos de generación, transmisión y distribución de saberes colaborativamente, tal como sucede en las redes sociales.

En tal sentido, los continuum para la inteligencia colectiva desde la coexistencia universidad-redes sociales se funda en la naturaleza reflexiva de la sociedad, por ende, expresa su capacidad de autorregulación y conformación organizativa como condición de existencia. Se traza un círculo virtuoso de continuum, cuyos resultados son insumos y producto al mismo tiempo, toda esa multidimensionalidad de autonomías y dependencias permitieron el acercamiento, contextualización, conceptualización y relevancia de las circunstancias de estudio, mediante un entramado de referentes teóricos y de experiencias de las que se desentrañan las cualidades que arrastran las causalida- des recíprocas que emergen de las interrelaciones dialógicas de autores socioeducativos e investigadores. Desde esta dinámica de reflexividad se perciben los significados que dan cuenta de cómo se hacen las cosas en la universidad, o cómo se capta su perfil organizacional, así como los significantes que expresan el desempeño deseado o performatividad que integra las diferentes perspectivas, a partir de las cuales se genera un círculo hermenéutico permanente para la comprensión e interpretación de la inteligencia colectiva desde la coexistencia universidad-redes sociales.

\section{ConTINuUм I Y II}

\section{Acercamiento, conceptualización y relevancia de las circunstancias de estudio}

Este continuum constituye el devenir que se construye para comprender el entramado teórico-vivencial que corresponde al acercamiento, conceptualización y relevancia de las circunstancias de estudios, la multiplicidad de coexistencias que surgen a partir de diferentes escenarios y perspectivas relacionadas con la praxis universitaria. Esto en cuanto a la generación, transmisión y distribución de conocimiento como razón de ser de las universidades y las implicancias de la hipermediación tecnológica en la configuración organizacional en red.

De allí que sus componentes constituyen el eje medular para develar la interrelación que existe entre la universidad y las redes sociales, como bases preeminentes de su coexistencia. En tal sentido, se parte de la consideración de Levy (2004), al referir que el contexto de las sociedades contemporáneas y sus organizaciones no se constituye 
únicamente por el territorio geográfico, sino que incluye "un espacio invisible de conocimientos, de saberes, de potencias de pensamiento en cuyo seno nacen y se transforman las cualidades de ser y maneras de actuar en sociedad".

En este acontecer los organigramas del poder pierden su resonancia y relevancia, al igual que las fronteras geográficas y disciplinarias para dar paso a un espacio multidimensional, donde la humanidad mediante interrelaciones dinámicas se encuentra y se produzca a sí misma en una temporalidad complejizante de orden transversal, plural e indeterminada.

Por este motivo, las interrelaciones que subyacen a esta realidad se han de concebir desde el conjunto de la sociedad, hacia ella misma y en potencia desde este conglomerado a cualquier punto $\mathrm{u}$ orden social que se vincule con cualquier otro. Según Morin (2002) esto se vislumbra mediante una interacción reticular y recursiva que esboza una espiral autopoiética de la existencia.

Desde esta perspectiva la universidad y las redes sociales, como religue simultáneo de organización, proceso y acción, dejan de estar segmentadas y separadas para constituirse en un devenir que se organiza permanentemente mediante una espiralidad reflexiva de orden emergente, generada por las autonomías y dependencias, que surgen por la confluencia reticular de flujos-reflujos de saberes, los cuales emergen del ecosistema producido por la hipermediación tecnológica y su movilidad discursiva.

Esta coexistencia busca potencializar mediante una gestión estratégica el diálogo de saberes y los procesos de conocimiento que se desprenden de éste, acordes a las nece- sidades sentidas del entorno social, cultural, tecnológico, económico y político, que le dan origen multidimensionalmente en lo local-global.

A este respecto, Tünnermann (2006) considera que se ha generado en el mundo una progresiva ampliación de la integración e interdependencia entre los distintos sectores sociales, por el incremento cualitativo y cuantitativo de la circulación de los flujos de información, por lo que desde entonces se ha venido avanzando hacia una sociedad en la que el conocimiento y su transmisión ocupan un papel cada vez más central en la dinámica socioeconómica.

De igual manera, a tono con este argumento, plantea que la valoración de los impactos generados en la Conferencia Mundial de Educación Superior en 1998 (CMES-1998), donde se realizó una gran movilización intelectual a nivel mundial de los actores involucrados en el quehacer universitario, el cual constituye un punto de partida obligado para la compresión de los cambios ocurridos y las perspectivas que persisten en la dinámica de las casas de estudio superior de América Latina y El Caribe, especialmente, porque estas consideraciones son el resultado de una agenda colectiva, cuya contribución perfila y recrea el espectro de actuación estratégica en este contexto.

Estas consideraciones se reconocen en la declaración de la UNESCO, en la CMES-2009, cuyos debates se presentan como: "la nueva dinámica de la educación superior y la investigación para el cambio social y el desarrollo", denominación que recoge de los debates, el carácter imperativo y estratégico que la universidad, en el desempeño de sus funciones primordiales: investigación, docencia y servicio a la comunidad, tienen que 
asumir para su transformación y de todos los niveles de enseñanza; de esta manera coadyuvar en el impulso y fomento del desarrollo sostenible de pueblos y naciones.

Es evidente que para lograr este cometido, las universidades, al igual que las organizaciones, grupos e individuos que conforman esta sociedad, tienen que buscar vías o mecanismos que germinen de su propia comprensión, para que se arraiguen y trasciendan a todos sus ámbitos de influencia, de manera tal que les permitan adaptarse, regularse y generar su transformación desde lo impredecible y perplejo de este acontecer.

Ahora bien, para percibir y comprender cualquier proyecto, proceso o acción de la dinámica organizacional universitaria, ineludiblemente se tiene que vislumbrar desde la heterogeneidad de sus interacciones y relaciones, las cuales se fundan en el lenguaje y la comunicación, ya que la interacción y comunicación son dos conceptos indisociables referidos a las relaciones sociales, a las coincidencias, perturbaciones y fluctuaciones que dentro del accionar del lenguaje y la expresión han intervenido en el desarrollo del ser humano a lo largo de su historia.

Según Maturana (1990) esta condición de existencia parte de la interacción de los componentes que se fundan en el lenguaje, las conversaciones y el diálogo, constituyéndose en génesis y transformación de la red de producciones que los produce cuando repercuten en el devenir de las relaciones sociales y la ética de la convivencia. Por su parte Luhmann (1998) refiere que sociedad y cultura deben su existencia a las acciones y retroacciones provenientes de la interacción social, pues el quehacer y la comunicación están intrínsecos en las interrelaciones, por lo que la sociedad es un conglomerado que se constituye en la movilidad discursiva, instaurada y entrelazada en la cultura.

Ahora bien, al trasladar esta consideración al acontecer actual surgen múltiples horizontes yuxtapuestos al efecto de redes, el cual se fundamenta en la relación multidimensional elemento-sistema en la dinámica reticular que no sólo ha modificado y ampliado la visión sobre las diversas posibilidades que se expresan en el acaecer organizacional contemporáneo, mediante los procesos de comunicación, sino que concurrentemente en este panorama de diversidad surgen ideas y sentimientos antagónicos que se entretejen y enredan de forma complementaria, producto de la más alta modernización y convergencia de tecnologías y las implicaciones culturales que esto conlleva en cuanto a la gestión de la información ilimitada y las posibilidades reales de su aprovechamiento.

En relación con lo expuesto, se refiere primeramente a la superación de la visión instrumentalista de los medios utilizados para la vinculación y comunicación, en cuanto a que dejaron de ser percibidos como un canal que transmite información, para ser considerados un espacio de encuentro y construcción de influjos sociales, un lugar donde según Scolari (2008) se expresan estrategias de acuerdos en consenso, efectos de dominación, tácticas de resistencia y construcción de identidades colectivas.

Insertado en esta reflexión se recrea un religue de procesos estratégicos, tácticos y operacionales con efectos en la construcción social-cultural que según este autor, y tomando la idea de Martín-Barbero (2003), permiten pasar "de los medios a las hipermediaciones", de esta manera, se pierde la 
particularidad del objeto para ganar en la interrelación de interactividades y procesos.

Por este motivo, al hacer referencia a la hipermediación tecnológica, no corresponde a un producto, una herramienta o un medio, sino a un conjunto de "procesos de intercambio, producción y consumo simbólico que se desarrollan en un entorno caracterizado por una gran cantidad de sujetos, medios y lenguajes interconectados tecnológicamente de manera reticular entre sí" (Scolari, 2008).

Este planteamiento expone con claridad lo que se asume en este estudio, en cuanto a la hipermediación tecnológica, es decir, a lo que encierra y enfatiza esta idea, la cual se focaliza en la trama de reenvíos, hibridaciones y contagios que la tecnología digital permite articular dentro del ecosistema mediático. Por ello descarta la consideración particular y desvinculada de los medios tecnológicos.

Dicho de otro modo, las hipermediaciones tecnológicas llevan a indagar en la emergencia de nuevas configuraciones que surgen por la autorregulación de actividades y procesos, así como la autoconformación organizacional, las cuales van más allá de los medios tradicionales.

La complementariedad universidad-hipermediación tecnológica tiene su fuente y origen en la vinculación comunicativa como proceso generador de interrelaciones e interacciones de los contextos que la proveen, existe, a su vez, en esta consideración procesos retroactivos y recursivos que se producen y complementan los unos a los otros en una dinámica dialógica complementaria, que según Morin (2002) permite en un mismo espacio de pensamiento la coexistencia de lógicas antagónicas, las cuales se necesitan para su producción y coexistencia.

Cuando se refiere a la comunicación, en estas condiciones, el propósito es ir más allá de las ideas clásicas y mecanicistas, enfatizando que se trata de procesos nolineales en tanto son multidimensionales, heterogéneos, aleatorios y azarosos, donde la evolución de la interacción comunicativa no es lineal, estática o sin matices, por el contrario, su progreso y desarrollo posibilitan la emergencia de redes interconectadas de relaciones.

En complemento a esta reflexión, tanto las relaciones como las interacciones comunicativas que las posibilitan se consideran como cambiantes, polisémicas, multifocales y de grandes matices interdinámicos, en otras palabras, es un proceso multidimensional de interacción-relación-comunicación, fundado en un rizoma polidialógico, del cual emana permanentemente un efecto complejizante.

Se constituye en un entramado fundado en la relación dinámica que emana de la acción comunicativa, entendida como una manera emergente de acción social en el entorno desarrollado. Este proceso estructura y articula la manera como los sujetos en una relación intersubjetiva perciben el mundo, al mismo tiempo que existe un redimensionamiento y reedificación en esta diversidad bio-psíco-social, ya que se encuentran en coexistencia, por lo que sus relaciones se dinamizan en un proceso de constante afectación recíproca, desde la heterogeneidad y multidimensionalidad de su acontecer.

Es así que las pautas y dinámicas del nuevo concepto de acción, organización y morfología social, tal como lo considera Castells 
(2006), gira en torno a la hipermediación tecnológica y lo que esta suscita en la reordenación de la temporalidad espacial, en cuyo orden intrínseco se redimensionan también los modos de interacción e interrelación a partir de los cuales confluyen procesos emergentes que afirman y privilegian los valores de libertad, autonomía, diversidad cultural, inclusión, equidad, participación, cooperación y solidaridad ecológica, como patrón y trama de esfuerzo colectivo para el bien común, de vivir y saber convivir.

En la contemporaneidad las redes sociales permiten captar estos movimientos de ideas y de valores mediante la colaboración y cooperación espontánea que subyace a su interactuar, cuyo fundamento esencial se constituye y percibe mediante un sistema abierto de construcción permanente, el cual involucra a conjuntos que se identifican mediante intereses comunes, necesidades y problemáticas, generando un intercambio dinámico entre personas, grupos e instituciones en contextos de complejidad, los cuales se organizan para potenciar los recursos que poseen de manera colectiva.

Imbricada en esta apreciación se encuentran los nuevos cometidos de las universidades, en las cuales se demandan, como cambios sustanciales, las estructuras académicas predominantes que responden a una concepción unidisciplinar del conocimiento $y$ que en momentos actuales se perciben como descontextualizadas y sin pertinencia social. En tal sentido se plantea "adoptar estructuras organizativas y estrategias académicas que les confieran un alto grado de agilidad y flexibilidad, así como la rapidez de respuesta y anticipación necesarias para encarar creativa y eficientemente un devenir incierto" (CRES-1996), tal como lo expresan los principios emanados de la Conferencia Regional de La Habana, realizada en 1996.

Mientras que en la CMES (1998) se proclama preservar y reforzar aún más las funciones básicas de educar, investigar y de extensión, para "contribuir al mejoramiento conjunto de la sociedad y al desarrollo sostenible, mediante la construcción de un espacio abierto que propicie la formación superior, para la cooperación de ciudadanos responsables y el aprendizaje a lo largo de la vida" (CMES, 1998). En cuanto a su accionar y pertinencia social señala que "deberían fomentarse y reforzase la innovación, la interdisciplinariedad y la transdisciplinariedad en los programas, fundando sus orientaciones en los objetivos y necesidades sociales y culturales" (CMES-1998).

El marco de acción estratégica para la transformación universitaria que surge de ambas agendas considera como pertinente una conformación organizativa abierta y flexible que admita la cooperación, participación, la inter y transdisciplinariedad de programas y acciones, con múltiples canales de vinculación para el fomento de los procesos de conocimiento e innovación de acuerdo a las necesidades de su entorno.

Subyacente a esta concepción se encuentran las organizaciones reticulares que según Bonifacio y colaboradores (2002) representan el enlace de complejas organizaciones distribuidas geográficamente, las cuales exigen configuraciones organizativas que integran arquitecturas abiertas, flexibles, de múltiples conexiones y diferentes vías de comunicación para el quehacer cotidiano y el aprendizaje organizacional. De allí que la coexistencia universidad-redes sociales enfatiza su relevancia como trama configurativa en red desde la interacción e 
interdependencia que se establece a nivel estructural y estratégico a partir de las relaciones dialécticas y sistémicas que permiten examinar constantemente las prácticas en su génesis y permanencia.

Ante este conjunto de pautas y lo que revierte en el monitoreo de acciones y retracciones, la homogeneidad organizacional está dando paso a la diversidad y heterogeneidad, donde la confluencia de nodos organizacionales, fomentados por la hipermediación tecnológica, conforman las organizaciones reticulares, de las cuales emergen nuevos significantes comunicacionales para el trabajo y las experiencias, así como en las diferentes y novedosas maneras de impulsar la invención, distribución y transmisión de conocimientos.

Desde las connotaciones de esta contextualización cabe preguntarse lo siguiente: ¿qué impacto generan estos aspectos en el ámbito universitario?, ¿cuánto ha evolucionado la estructura organizacional de la universidad como organización encargada de producir talento humano, de transferir y fomentar a las comunidades de influencia, la apropiación social del conocimiento?, ¿qué mecanismos utiliza para desarrollar capacidades de adaptación en contextos inciertos y cambiantes?

Estos interrogantes conducen a un cambio profundo en los procesos básicos, las actividades y toda su configuración organizacional. En este sentido Salinas (2004), Silvio (2004) y Brunner (2003) coinciden en que las universidades comienzan a reestructurarse y adoptar modalidades de coordinación y gestión para alejarse de la centralización de procesos, la toma de decisiones a nivel jerárquico y a aproximarse a topologías organizativas horizontales conformadas en red, donde los procesos operan como unidad en tiempo real a escala planetaria, para impulsar y potenciar el intercambio de saberes, su producción, almacenamiento, distribución y acceso a los fines de compartir y desarrollar de forma colectiva capacidades de creación, entendimiento y transformación de la realidad sobre la base fundamental de las relaciones humanas.

Ahora bien, partiendo del hecho que la educación constituye la base y el punto de partida para cualquier transformación de la humanidad y desde su seno se posibilita la movilización de acciones y procesos, a partir de los cuales pueden gestarse y pronunciarse la metamorfosis de nuestro estilo de vida. Pero también es cierto que aún persiste en su seno la manifestación de un pensamiento de orden lineal y fragmentado sobre el cual se tiene que actuar transdisciplinariamente para poder afrontar las circunstancias y situaciones de incertidumbre y ambigüedad que caracterizan la complejidad creciente de este acontecer.

Subyacente en esta situación existe una problemática a nivel socioeducativo, que se constituye como medio y fin para su transformación, como un bucle recursivo que se autorregula y autoproduce a sí mismo, en palabras de Pupo (2010), "el saber educativo no puede cambiar sin transformaciones profundas en la educación y ésta resulta infecunda sin una reforma en el pensamiento y en la praxis en que encuentra concreción".

Estos argumentos plantean otra manera de comprensión, una paralógica que necesariamente tiene que partir por el desaprendizaje y reaprendizaje que posibilita la reconceptualización y redimensionamiento del conglomerado universitario, principalmente, lo que concierne a sus procesos de gestión y 
comunicación, los cuales tienen que partir por repensar el conjunto de ocurrencias, acciones y circunstancias, involucradas en los proyectos que vislumbran el suceder de las casas de estudio superior, desde una nueva base de comprensión que no sólo considere la multiplicidad de la coexistencia que emerge de las interacciones e interrelaciones recíprocas de los componentes y el todo, sino que permita establecer puentes y vínculos entre lo particular y colectivo, así como también una metaestructura de pensamiento para captar las cualidades, significados y significantes que se movilizan a partir de las dialógicas complementarias y concurrentes inmersas en la praxis universitaria.

\section{El complexus de la coexistencia universidad-redes sociales}

Según Ander Egg (2001) el mayor desafío que enfrentan los grupos, organizaciones y sociedades en la actualidad es pensar desde la globalidad planetaria, ya que la obsolescencia y biodegradabilidad de las referencias estables son constatadas en corto tiempo. Por ende, es necesario suspender la representación de las certidumbres absolutas del determinismo y la linealidad, para aceptar y enfrentar la dinámica del relativismo y todos los riesgos que ello implica.

En atención a este argumento y lo expuesto sobre el devenir organizativo universitario y las redes sociales se comprende esta coexistencia como un conjunto de constituyentes heterogéneos inseparablemente asociados, donde se admite la dialógica del orden y desorden, así como la inseparabilidad del sujeto-objeto de la realidad en cuestión, lo cual denota su complexus o entretejido en conjunto, según Morin (1990) constituye la trama de eventos, acciones, interacciones, y retroacciones que conforman la realidad, de tal manera que permite percibir los rasgos de lo enredado, de la ambigüedad y la incertidumbre.

De esta manera, el complexus de la coexistencia universidad-redes sociales se presenta cuando ante demandas y desafíos externos, tal como la cultura multidimensional que aclaman la CRED-1996, la CMES-1998 y la CMES-2009, se activa la sinergia entre sus diferentes componentes, lo cual origina un torbellino de eventualidades y efectos que generan estados para la producción de otros, estos a su vez interpelan una serie de acciones y retroacciones a niveles particulares por la interrelación, concatenación e interdependencia de sus elementos, los cuales producen la interretroacción, para el funcionamiento y cumplimiento de su responsabilidad social. Este interactuar permite la adecuación organizacional al producir por sí misma su autorregulación.

En este orden de consideraciones, cuando la lógica de la praxis organizacional llega al punto donde todos los componentes se activan para atender el requerimiento, se produce un encuentro dialéctico de causalidades múltiples y extensivas o mega-causalidad que permite la configuración de entornos organizativos autoformativos o inteligentes, mediante la transretroacción, aludiendo que la acción de distribuir el conocimiento que permite regular el sistema académico y administrativo al interior de las universidades, pero también al consolidar las redes sociales con otros contextos organizacionales se trasciende al exterior de las casas de estudio mediante un flujo simultáneo, complementario y recursivo de saberes.

De manera concurrente este proceso de transretroacción genera un efecto de espiral 
reflexiva que fomenta la coexistencia multidimensional en una retícula de producción informacional donde cada dependencia y/o actor universitario no sólo interactúan de forma natural en pro de la gestión distribuida del conocimiento, sino que se nutren unos de otros para reforzarse mutuamente.

Lo esgrimido anteriormente constituye un sistema referencial de aspectos que permiten percibir y comprender las relaciones que emergen de la coexistencia de universidad-redes sociales, donde cobra fuerza el siguiente cuestionamiento: ¿qué relaciones, intercambios, interdependencias y acciones se han de repensar en la trama teórico-conceptual para la generación y concreción de una reflexividad sistémica para la conducción estratégica de la coexistencia universidad-redes sociales?

En tal sentido se plantea generar la comprensión de las categorías que emergen de los hallazgos en el entorno universitario relacionados con la gestión universitaria en el marco de las redes sociales para captar desde el significado que le atribuyen los actores socioeducativos al redimensionamiento del vínculo social en el espacio multidimensional del ámbito universitario y las redes sociales, de aquí la intencionalidad de generar una reflexividad sistémica que perfile las bases teórico-conceptuales para la gestión de la coexistencia multidimensional universidad-redes sociales.

\section{Relevancia contextual de la indagación}

Esta indagación está enfocada en una reflexión teórico-conceptual para la gestión de la coexistencia universidad-redes sociales, como base gnoseológica de su organización y conducción, se presenta como altamente justificable pues supone el tratamiento de una temática de especial relevancia tanto para los espacios internos de las universidades como para las organizaciones sociales en general.

En sintonía con lo expuesto, la preponderancia de la temática en estudio radica en la posibilidad de propiciar un conjunto de transformaciones esenciales, capaces de reestructurar la visión y misión de la universidad, no sólo como ente generador de conocimientos socialmente pertinentes, sino como centro reticular o red de redes para la distribución de conocimiento pertinente, lo cual indudablemente generará beneficios de múltiple alcance en la calidad y conveniencia de la producción intelectual científica y tecnológica de la universidad sobre la base del conocimiento distribuido, compartido y/o transferido, equitativa y oportunamente.

Desde esta perspectiva se privilegia las demandas de una educación con sentido complejo que también refiere a las redes sociales como cosmovisión o metaestructura de pensamiento que admite las comprensiones mutuas en todos los sentidos, niveles educativos y en todas las edades, para suscitar la capacidad de repensar los problemas humanos desde la sistematicidad de su interactuar y relacionar. Así como la multidimensionalidad que envuelve el acontecer de los fenómenos con su realidad, tomando como fundamento de transformación los rasgos que caracterizan la sociedad de red: flexibilidad, adaptabilidad, escalabilidad y capacidad de supervivencia.

En cuanto al abordaje de la praxis y la gestión de la multiplicidad educación-redes sociales, permite integrar las diversas posibilidades heurísticas que surgen en torno a las 
redes sociales y lo que presupone un acercamiento a la transformación universitaria desde la posibilidad de cambio que tiene un sistema como condición de autopoiesis organizativa.

\section{Continutum III y IV}

\section{El sendero epistemológico y la elucidación de las circunstancias de la praxis universitaria}

\section{Consideraciones previas}

El sendero epistemológico y procedimental de esta indagación constituye un recorrido que se construye en y para la prosecución de su intencionalidad, cuya búsqueda abarca y se focaliza en la misión que le corresponde cumplir a la universidad de este tiempo, así como los procesos de gestión que se han de suscitar en su seno, para asumir adecuadamente el fenómeno de las redes sociales, especialmente, cuando su responsabilidad social se recrea en una retícula de relaciones fundadas en la educación, investigación y extensión, que interactúa con y sobre los individuos y sus intersubjetividades, razón por la cual hace eco de la diversidad en su generalidad unificadora y en la singularidad de cada sujeto.

Es por ello que para poder llevar a cabo el discurrir investigativo fue necesario examinar los patrones de pensamiento, a objeto de repensar lo pensado desde una nueva comprensión que permita establecer puentes de encuentro y de cohesión entre individualidades y colectivos para distinguir, vincular y afrontar lo incierto y perplejo que sirve de antídoto para el pensamiento único y segmentador. De esta manera se desarrollaron capacidades adecuadas para percibir los nexos invisibles que coexisten en la trama de relaciones de la organización universitaria y su contexto, en tal sentido se asumió una postura epistemológica imbricada en las ciencias de la complejidad, soportada en los criterios de Bertalanffy (1968), Bateson (1976), Foerster (1988), Maturana y Foerster (1988), Watzlawick (1990), Jutoran (1994), Morin (1999, 2002), Brugger y colaboradores (2003), así como Delius, Gatzermeir y otros (2005).

En consecuencia, esta investigación se vivifica en la trama invisible de multiplicidad sistémica, como condición de evolución y transformación del escenario socioeducativo universitario y de sus actores, partiendo de la consideración de aceptar el despliegue de la heterogeneidad y la multidimensionalidad de las relaciones y circunstancias de su quehacer.

\section{Construcción metódica}

En cuanto a la configuración metódica se sustenta en un bagaje ontoepistémico que permite buscar, percibir y comprender en la panorámica que involucra la gestión de una multidimensionalidad compleja como lo es la coexistencia universidad-redes sociales, lo que concierne a sus vínculos como realidad fluctuante, aleatoria y difícilmente predecible, lo que refiere a las posibilidades de coexistir desde su propia dialógica.

Dicha perspectiva teórica, conceptual y epistemológica de saberes supone ante todo un cambio dirigido a vencer la tentación de la rutina, de la simplificación y la superficialidad, del determinismo mecanicista y de la inercia, de la repetición acrítica de los mismos esquemas mentales y prácticos que recaen sobre la comunidad universitaria. 
En tal sentido se provee un movimiento recursivo de de-construcción y construcción para captar las potencialidades que tienen los fenómenos emergentes, los errores, el despliegue de las subjetividades individuales y colectivas, con toda la espontaneidad y experiencia singular que ellas portan, la riqueza de la cotidianeidad y sus redes informales en los nuevos escenarios educativos y en sus contextos, así como los nuevos valores y saberes relativos a la capacidad de autoorganización e interacción de los grupos que en ellas surgen constantemente.

Para lograr esta intencionalidad investigativa se parte de los postulados de la cibernética de segundo orden o reflexión sobre la reflexión (Foerster, 1988), en complementariedad con los principios para un pensar complejo (Morin, 2002) a partir de los cuales se conformó una reflexividad multifacética, bajo un enfoque inter y transdisciplinario, que permitió captar el escenario investigativo mediante un proceso activo, recursivo, cíclico, creativo y flexible, donde se da relevancia a las relaciones sociales, al sentido y sentimiento que estas movilizan.

En cuanto a los actores socioeducativos que proveen la información que le da significado al estudio se seleccionaron cuatro relatores por el rol que desempeñan y su experiencia en el ámbito universitario, así como en la conducción de procesos de conocimiento en la universidad de este tiempo. Esto adquiere pertinencia por los cargos que desempeñan como directivos y docentes de las casas de estudio de la Universidad Nacional Experimental Rómulo Gallegos, donde se seleccionó al decano y director del área de Ingeniería de Sistemas. Mientras que la Universidad Nacional Experimental Simón Rodríguez a su coordinador de posgrado. En el sector empresarial, la Hidrológica Páez por su condición de empresa mixta (pública y privada), su gerente de comercialización permite dar cuenta de las fuerzas vivas de la región.

La valoración de la información se da en el mismo momento de captar un evento desde diferentes puntos de vista, de vivir la realidad estudiada y comprenderla en su propia dinámica. Asimismo, se aplica la triangulación constante a partir de la interrelación de dialógicas provenientes de diferentes fuentes, en este caso informantes clave-teóricos-investigadores.

Así, se despliega un proceso cíclico de continuo preguntarse y comparar para la compresión e interpretación, utilizando la categorización, subcategorización, organización y sistematización de la información hasta su saturación, lo cual permite desentrañar las cualidades que arrastran las causalidades recíprocas que emergen de las interrelaciones dialógicas de autores, informantes clave e investigadores, guiadas por un conjunto de temas que permitieron el diálogo de saberes desde donde se dilucida la realidad onto-epistémica de la coexistencia universidad-redes sociales, mediante significados que dan cuenta del perfil organizacional, o cómo se hacen las cosas en la universidad. De igual manera en esta dinámica de reflexividad se perciben los significantes que expresan el desempeño deseado o performatividad de acuerdo con la perspectiva de los actores socio-educativos.

De este modo, atendiendo a lo planteado, la construcción metódica se erige en una circularidad cognitiva que progresivamente gira alrededor de sí misma, para redefinirse en el ascenso y descenso del continuum de otra circularidad de saber, de la cual se 
hace partícipe como generatividad y transformación al mismo tiempo de compresión e interpretación, tal cual una espiralidad multireflexiva desde donde se genera un círculo hermenéutico permanente para la elucidación. Este fluir cognitivo concurrentemente es génesis y transformación, a partir del cual se traza un sendero propio, cuyo recorrido permite redefinir los conceptos y métodos tradicionales para construir desde las interrelaciones de la propia vivencia, de su praxis y de sus intersubjetividades, el recorrido epistemológico por medio de las categorías de sentido que emergen como un nuevo orden innovador.

\section{Continuum IV y V}

\section{Develando significados onto-epistémicos de la praxis universitaria}

En correspondencia a lo antes planteado, especialmente, para la consecución del propósito fundamental de este estudio, se recurrió a una espiral hermenéutica de ascenso y descenso para la captación, sentido y conocimiento del ser-existencia, como lo es la génesis de reflexividad sistémica para la gestión de la coexistencia universidad-redes sociales, a partir de las interrelaciones dialógicas inmersas en la praxis universitaria, en tal sentido se recurre a un entramado de accionesretroacciones, las cuales superan el orden y secuencia que impone el método lineal, mediante un fluir entretejido, donde insumos y resultados se complementan mutuamente para percibir a partir de la inteligencia colectiva, conformada por los autores, informantes clave e investigadores, la realidad ontoepistémica de la praxis universitaria.
En la interrelación dialógica generada entre la universidad y los temas seleccionados para comprender la praxis universitaria, se escoge la dimensión de gerencia universitaria porque constituye el punto medular de esta indagación, donde convergen cinco vertientes argumentativas que recogen los diez temas que permitieron concebir el diálogo de saberes entre informantes claveteóricos-investigadores, los cuales son: la hipermediación tecnológica y la sintonía que tiene la universidad con la sociedad del conocimiento, la gestión distribuida en contextos universitarios, las redes sociales, la cultura para la diversidad y el aprendizaje permanente, así como los procesos de conocimiento que comprenden: acceso, almacenamiento, transferencia, distribución y generación de nuevos saberes.

Es de hacer notar que cada una de estas vertientes movilizan una multidimensionalidad de condiciones y cualidades, mediante categorías y subcategorías que se entrelazan rizomáticamente para el confluir de un conjunto de argumentos provenientes de las apreciaciones de los informantes clave, sobre la conducción estratégica de la coexistencia universidad-redes sociales, de cuales emergen significados referentes al perfil organizacional, así como significantes para el desempeño esperado o performatividad.

Tanto la interrelación de dialógicas como la trama de subcategorías subyacentes derivadas de los relatos de los informantes clave, se integran en una sinopsis de interrelación dialógica para percibir en detalle el devenir de las concatenaciones y dependencias que se generan en el interactuar universitario, las cuales conciben a su vez, la red de categorías y subcategorias subyacentes en la praxis universitaria, así como su realidad 
onto-epistémica, cuya integración permite conformar la matriz de demandas y desafíos, así como los entramados rizomáticos de saberes, referentes a la disposición del perfil organizacional, así como a la performatividad en contextos universitarios reticulares.

\section{Autopoiesis ORgANizACIONAL PARA LA GÉNESIS Y \\ TRANSFORMACIÓN \\ DE LA COEXISTENCIA \\ MULTIDIMENSIONAL \\ UNIVERSIDAD-REDES SOCIALES}

Subyacente en lo que movilizan los procesos de compresión-interpretación, especialmente, lo que refiere a las demandas y desafíos que enfrenta la universidad de este tiempo para su adecuación organizativa, así como las habilidades y competencias que se tienen que alcanzar para lograr criterios que acrediten su performatividad y transformación hacia el desempeño deseado. Surge un conjunto de consideraciones que perfilan el cuerpo teórico conceptual para la gestión de la coexistencia multidimensional universidad-redes sociales.

Es evidente que en el escenario planteado todos los elementos constituyentes del sistema organizacional universitario comparten responsabilidades que conllevan a la retroactividad múltiple generada en el conglomerado social, por ello, el motor dinamizador lo constituye el incesante proceso de retroalimentación que se realiza recursivamente mediante las acciones y retroacciones generadas en la dinámica de dialógicas concurrentes y complementarias a niveles inter y transorganizacional, las cuales propagan por todo el ámbito organizativo la autorregulación y la autoconformación cuando así lo amerita.
Esta consideración constituye el fundamento de la autopoiesis organizativa universitaria, donde las intertransretroacciones hacen posible el eslabonamiento de dependencias y actividades derivadas del interactuar, especialmente lo que concierne a espacios o escenarios de cooperación, participación y cognición fundadas en relaciones de confianza, entendimiento y consenso, necesarias para revelar la creatividad y la innovación como requerimiento ante la ambigüedad y complejidad de la estructuración coetánea, y a su vez mediante la capacidad regenerativa reticular de la transretroacción promover nuevos patrones organizacionales.

\section{ReFleXividad SISTÉMICA PARA LA GESTIÓN EN CONTEXTOS UNIVERSITARIOS RETICULARES}

Anclada a la consideración de autopoiesis organizacional desde la retroalimentación propagada se vislumbra un proceso de aprendizaje permanente en el ámbito universitario que articula las concepciones del conocimiento individual y organizacional mediante procesos de retroactividad múltiple generados por la interrelación de los componentes del sistema organizacional. La movilización que esta dialógica conlleva manifiesta la permanente transformación recíproca que sucede en un ciclo espiralado de ascenso y descenso, según sean sus demandas y desafíos.

Es así que el proceso de gestión se redimensiona sobre la base de la noción que el conocimiento parte de la mente individual, pero se funda en las relaciones sociales, en tal sentido la organización, según Sisto (2004) es entendida como un conjunto de articulaciones y rearticulaciones que fluyen 
mediante procesos comunicativos, migrando de individuo a individuo, de grupo en grupo y de organización en organización.

Entonces, tomando en cuenta la emergencia de lo complejo, la autopoiesis como principio de existencia, la equifinalidad como propiedad de alcanzar la meta por diversas rutas y los isomorfismos como noción de consenso y alianza entre diferentes lógicas de ordenamiento, surge la performatividad, como patrón estructural y configurativo de gestión distribuida en contextos universitarios deslocalizados, apuntalada en procesos de conocimiento, sobre la base de las interretrotransacciones que suceden por la retroactividad múltiple del conglomerado organizacional y las posibilidades estratégicas que conlleva el discurso.

\section{INTELIGENCIA COLECTIVA DESDE LA COEXISTENCIA UNIVERSIDAD- REDES SOCIALES}

Sobre la base sinóptica de las consideraciones expuestas, por un lado, el discurso moviliza las vías intersubjetivas inmersas en los acuerdos consensuados y por el otro, la performatividad comprende sus posibilidades estratégicas en la conducción del conglomerado organizacional, estas dos concepciones producen una condición bidireccional que perfila el accionamiento universitario, pero al entrar en contacto o al ser asimilada reflexivamente mediante los esquemas de conectividad entre individuos, grupos y organizaciones, se transforma en una condición tridimensional que refiere a un holograma, que admite el movimiento y el fluir de manantiales de saberes que se alternan mediante la interferencia multidireccional, convergente y divergente para el refuerzo constructivo de los proyectos y procesos de la trama configurativa.

En este sentido, el accionamiento traslada a su esencia el rasgo connotador de la totalidad, o dicho de otra manera, los constituyentes tienen significancias del todo conformador, como un sello o rasgo principal que perfila la identidad o consonancia a cumplir independiente en los diferentes nodos de la retícula.

Otro aspecto onto-epistémico que cobra fuerza en la gestión de contextos universitarios reticulares, es el referente a la conformación hologramática conjugada a la convergencia, acceso y bifurcación de interrelaciones que no sólo permiten percibir la relación de estrecha inseparabilidad del sistema organizacional y sus componentes, sino que también trae consigo la recursividad de las intertransretroacciones que éste establece con su contexto global.

De esta manera, los aspectos planteados abren la posibilidad de una relación asociativa entre contenidos de información que van conformando un recorrido no lineal, ni único, sino de múltiples y diversas conexiones, conforme al funcionamiento de la mente humana, que conmuta instantáneamente al dato siguiente, según sea sugerido por asociación de ideas, siguiendo la intrincada trama de caminos de la retícula organizacional que refiere a una inteligencia que parte de la vinculación e interrelaciones multidireccionales, a modo de hipertextos y recursividad, las cuales refieren redes de interacciones continuas, conectividad, bifurcaciones, nexos, diversas vías de acceso, de acción y retroacción, de retículas de información y conocimiento sin fronteras, donde la gestión del conocimiento es infalible en cualquier conglomerado sociocultural. 


\section{LA ESPIRALIDAD REFLEXIVA PARA LA GESTIÓN DE LA COEXISTENCIA UNIVERSIDAD- REDES SOCIALES}

Para vislumbrar y significar los procesos de conducción de la coexistencia multidimensional desde la praxis universitaria, se esbozan tres configuraciones circularmente concatenadas con igualdad de correspondencia, como anillos abiertos que se enlazan de acuerdo a las circunstancias, a partir de las cuales emerge la espiralidad reflexiva desde tres perspectivas que fluyen en una multidimensionalidad recursiva, lo micromeso, meso-macro y macro-meta.

Circularidad de interrelaciones micro-meso o de actividades, donde los actores socioeducativos conectan sus acciones, los conceptos, teorías, símbolos e historias a procesos de conocimiento continuo, lugar en el que se recurre al consenso, a la cooperación y al apoyo mutuo para la conducción de proyectos y procesos en aprendizaje continuo.

Circularidad de interrelaciones meso-macro o de performatividad, es donde la actividad discursiva es recibida en un período o contexto en el cual adquiere sentido, sucede cuando la intersubjetividad le confiere voz y acción, hace referencia en un avance cíclico, al recurso estratégico de las prácticas discursivas de individuos, grupos y organizaciones que trazan mapas y senderos de conocimiento en correspondencia con la potencialización de las actividades básicas de investigación, educación y extensión, tanto intra como transorganizacionalmente en función de la atención y reforzamiento de las dimensiones de acceso-generación, transmisión-distribución e innovación-creatividad.
Circularidad de interrelaciones macro-meta o de conectividad, en este circuito se integran los circuitos de actividad con las dimensiones performativas en una disposición que se ordena a similitud de una estrella de cinco puntas en analogía a la estrella de mar, cuyas partes conformadoras son autónomas y tienen la capacidad de regenerarse a partir de cualquiera de ellas, en el caso de suceder algún daño, esto es admisible por las características que tiene la configuración reticular, de igual manera al unirse la actividad y performatividad, se asumen acuerdos y afirmaciones para la acción, de los cuales emergen nuevas posiciones subjetivas que caen en un nuevo circuito de actividad y performatividad que también genera prácticas organizacionales en constante aprendizaje para dinamizar el talento e inteligencia para la innovación.

\section{CONSIDERACIONES FINALES}

Para posibilitar la coexistencia multidimensional universidad-redes sociales, así como su gestión, se presentan como consideraciones finales un conjunto de posibles rutas, alternativas, escenarios y recursos que se acomodan de acuerdo a cinco referentes: ontológico, axiológico, epistemológico, metodológico o referido a los sistemas y teleológico referido a las personas.

Desde el referente epistemológico, las universidades tienen que recorrer diferentes estadios organizacionales que le permitan instaurar una cultura fundada en saberes, esto corresponde a una acertada gestión del conocimiento para posibilitar su tránsito desde una base cognitiva caótica, luego pasar a ser una organización consciente de su conocimiento, hasta estar equipada 
y basada en procesos de reflexividad permanente.

El referente axiológico, constituye la guía para que la universidad sea un contexto organizacional basado en conocimientos, por ello se requiere la reconstrucción organizacional en la acción conjunta del conglomerado para configurar el imaginario ético fundado en la libertad, autonomía, inclusión, equidad, cohesión, cooperación, solidaridad ecológica y participación, cuya base de activación gira en torno al reforzamiento permanente de sus funciones básicas, así como propulsar de manera colectiva escenarios estratégicos que permitan la excelencia organizacional.

El referente ontológico constituye las búsquedas que se realizan en la praxis organizacional para su constante renovación, en tal sentido cobra fuerza el planteamiento de esta reflexividad, es decir, percibirse desde las redes sociales en pertinencia total con su contexto, desde donde se autorregula y autoconforma en una trama de esfuerzo colectivo, de intercambio dinámico y cooperación espontánea entre personas, grupos e instituciones.

\section{REFERENCIAS}

Ander-Egg, E. (2001). Acerca del conocimiento $y$ del pensar científico. Buenos Aires: Grupo Editorial Argentina.

Bateson, G. (1976). Pasos para una ecología de la mente. Buenos Aires: Ediciones Carlos Lohl.

Bonifacio, M., Bouquet, P. \& Cuel, R. (2002). The Role of Classification(s) in Distributed Knowledge Management. To appear in
El referente teleológico, o dirigido a las personas, desde sus relaciones constituye la potencialidad para que algo exista y se base en criterios de entusiasmo, confianza y capacidad de aprendizaje permanente, estas son según Morin (2002) y Senge (2006) las siguientes: pensamiento sistémico, dominio personal, modelos mentales, construcción de una visión compartida y aprendizaje en equipo.

El referente metodológico o dirigido a los sistemas, requiere la conducción a gran escala de conjuntos organizacionales conformados por grupos de redes virtuales, unidos según los intereses específicos de cada uno, es necesario prever un recorrido que comprenda herramientas, procesos, estadios y funciones que promuevan la gestión distribuida del conocimiento en contextos universitarios conectados en red, desde esta perspectiva es evidente que la hipermediación tecnológica constituye el soporte indispensable para apoyar la creación y conducción de configuraciones autónomas deslocalizadas, de allí la importancia de contar con componentes que garanticen la producción, acceso, almacenamiento, transmisión y distribución de los saberes que se generan local y globalmente.

the Proceedings of 6th International Conference on Knowledge - Based Intelligent Information Engineering Systems \& Allied Technologies, Special Session on Classification. Italy: IOS Press.

Brugger, et ál. (1983). Diccionario de filosofía. Barcelona: Editorial Herder.

Brunner, J. (2003). Educación e internet la próxima revolución. Chile: Brevarios Fondo de Cultura Económica. 
Castells, M. (2001). La Galaxia Internet. Reflexiones sobre internet y sociedad. Barcelona: Areté.

Castells, M. (2006). La sociedad red, una visión global. Madrid: Alianza Editorial.

Deleuze, G. \& Gauttari, F. (2000). Rizomas. Valencia: Editorial Pre-textos.

Foerster, H. (1988). Construyendo una realidad. En: Watzlawick, P., et ál. La realidad inventada. Buenos Aires: Gedisa.

Jutoran, S. (1994). El proceso de las ideas sistémico-cibernéticas. Argentina: Instituto de Terapia Sistémica.

Levy, P. (1999). ¿Qué es lo virtual? Barcelona: Ediciones Paidós.

Levy, P. (2004). Inteligencia colectiva por una antropología del ciberespacio. Recuperado desde [http://inteligenciacolectiva.bvsalud.org/channel. php?lang=es\&channel=8].

Luhmann, N. (1998). Sistemas sociales: lineamientos para una teoría general. Barcelona: Editorial Anthropos.

Martin-Barbero, J. (2003). De los medios a las mediaciones, comunicación, cultura y hegemonía. Bogotá: Editorial Nomos.

Martínez, M. (2002). Ciencia y arte en la metodología cualitativa. México: Editorial Trillas.

Maturana, H. (1990). Seminario Conversando con Humberto Maturana. Buenos Aires: Instituto de Terapia Sistémica.

Maturana, H. \& Foerster, H. (1988). Biología, cibernética y comunicación. Buenos Aires: Asociación Sistémica.

Morin, E. (2002). Los siete saberes necesarios para la educación del futuro. Buenos Aires: Nueva Visión.

Morin, E. (2002). Educar en la era planetaria. España: Gedisa.
Morin E. (1999). La cabeza bien puesta. Repensar la reforma, reformar el pensamiento. Argentina: Nueva Visión.

Nederr, I. (2006). Estrategia de estudios a distancia. Modalidad mixta. Caso área de Ingeniería de Sistemas de la Universidad Rómulo Gallegos. Simposio Internacional de Educación a Distancia: Universidad Bretaña Sur de Francia y Universidad Rómulo Gallegos, Venezuela.

Nederr, I. (2006). Las instituciones universitarias de carácter experimental en la sociedad del conocimiento. El momento de la E-Universidad desde la gestión del conocimiento. Trabajo para optar al escalafón de Agregado en la Universidad Nacional Experimental Rómulo Gallegos. San Juan de los Morros, Estado Guárico.

Nederr, I. (2007). La universidad electrónica: una visión de organización inteligente desde la gestión distribuida del conocimiento. Tesis doctoral. Universidad Nacional Experimental Rómulo Gallegos.

Nederr, I. (2009) La gestión distribuida del conocimiento desde la transretroacción en entornos universitarios reticulares. Tesis doctoral. Universidad Nacional Experimental Politécnica de la Fuerza Armada Nacional-UNEFA.

Pupo, R. (2010). Curso taller: el ensayo como búsqueda y creación. Hacia un discurso de aprehensión compleja. Multidiversidad Mundo Real Edgar Morin. Recuperado desde [http://www.encuentrosedgarmorin.org/cursoensayo].

Scolari, C. (2008). Hipermediaciones. Elementos para una teoría de la comunicación digital interactiva. Barcelona: Gedisa.

Senge, P., et ál. (2006). La quinta disciplina en la teoría y práctica. Estrategias para cons- 
truir la organización abierta al aprendizaje. Barcelona: Granica.

Sears, F. \& Zemanski, M. (1971). Física. Adison Wesley. Madrid: Ediciones Juan Bravo.

Silvio, J. (2004). La virtualización de la universidad. ¿Cómo transformar la educación superior con tecnología? España: Instituto Internacional de la UNESCO para la Educación Superior en América Latina y El Caribe -IESALC.

Sisto, V. (2004). Teorías organizacionales postmodernas y la gestación del sujeto postmoderno. Tesis doctoral. Barcelona: Universidad Autónoma de Barcelona.

Tünnermann, C. (2006). Universidad, pertinencia, calidad y acreditación en un mundo globalizado. GUNI. Discurso inaugural de la 3á. Barcelona: Conferencia Internacional de Barcelona.

UNESCO (1996). Conferencia Regional sobre Políticas y Estrategias para la Transforma- ción de la Educación Superior en América Latina y El Caribe. La Habana: Palacio de las Convenciones Ciudad de La Habana.

UNESCO (1998). La educación superior en el siglo XXI. Visión y acción. En: Conferencia Mundial sobre la Educación Superior. París.

UNESCO (2009). La nueva dinámica de la educación superior y la investigación para el cambio social y el desarrollo. En: Conferencia Mundial sobre la Educación Superior. París

Valencia, C. \& Gatzermeir, M. (2005). Historia de la filosofía. Desde la antigüedad hasta nuestros días. Alemania: Könemann. Tandem Verlag GmbH.

Von Bertalanffy, L. (1968). Teoría general de sistemas. México: Fondo de Cultura Económica.

Watzlawick, P. (1990). Lo malo de lo bueno. Barcelona: Editorial Herder. 